\title{
Liste des portraits montrés dans le livre
}

Figure 1 Andreas Möller, Marie-Thérèse à l'âge de onze ans, vers 1727, 94 x $75 \mathrm{~cm}$, Ambras, Kunsthistorisches Museum Vienne, Gemäldegalerie, Inv. Nr. GG_2115, Autriche, (ㅇ «KHM-Museumsverband »- $\mathbf{8 0}$

Figure 2 Attribué au peintre Anton von Maron, mais provient probablement d'un autre peintre autrichien, Marie-Thérèse veuve en fondatrice de l'Ordre des Dames nobles d'Innsbruck, après 1765, 230 x $161 \mathrm{~cm}$

(c) Burghauptmannschaft Österreich, Sammlung:

Bundesmobilienverwaltung, Hofburg Innsbruck, MD 060828/000, Autriche, Photo: Bunge (Firma Neubauer) $-\mathbf{8 7}$

Figure 3 Imitation de Martin van Meytens, Marie-Thérèse, années 1740, 140 × 103 cm, Inv. Nr. S/841, Musée régional de Celje, Slovénie — 106

Figure 4 Franz Anton Palko, Marie-Thérèse, 1756, $265 \times 148 \mathrm{~cm}$, abbaye de Lilienfeld, Autriche, Stiftsarchiv, Stift Lilienfeld, Gemäldesammlung - 108

Figure 5 Daniel Schmidelli, ou Schmidely, Marie-Thérèse en roi de Hongrie, 1742, $237,5 \times 157 \mathrm{~cm}$, Inv. Nr. A 133, Collection of Bratislava City Gallery, Palais Primatial de Bratislava, Slovaquie. Galéria mesta Bratislavy — 111

Figure 6 Johann Baptist Glunck ou B. Ilye Glunck ? Marie-Thérèse en roi de Hongrie, huile sur toile, $248 \times 154$ cm, 1753, Accession Number : Gy/1046 (MG 4196), aujourd'hui au château de Fertőd en Hongrie. Eszterháza Kulturális, Kutatóés Fesztiválközpont : Közhasznu Nonprofit Kft. (Eszterháza Cultural, Research and Festival Centre Public Benefit Non-Profit LLC.)

(C) Photographe: Miklós Sulyok — 114

Figure 7 Artiste originaire d'Europe centrale, Marie-Thérèse, années 1740, 84,8 x 67,4 cm, Nr. MK 10251, château de Kežmarok, Foto Muzeum v Kežmarku, Slovaquie - 116

Figure 8 Artiste originaire d'Europe centrale, Marie-Thérèse, années $1750,85,5 \times 70 \mathrm{~cm}$, Nr. MK 52, château de Kežmarok, Foto Muzeum v Kežmarku, Slovaquie — 117

Figure 9 Hieronymus Doffy, portrait de Marie-Thérèse réalisé entre 1745 et 1750, 130 x 108 cm, Inv Nr K 1900/28, Hôtel de ville de Bruxelles, Musée de la Maison du Roi, Collection des Musées de la ville de Bruxelles, Belgique - 121

Figure 10 Matthias de Visch, Marie-Thérèse, 1750, 274 x 204 cm, Inv. Nr. 0000 GRO 0451.I, Hôtel de ville de Bruges, Belgique, Source: Musea Brugge, Lukasweb.be-Art in Flanders, photo: Hugo Maertens, Musea Brugge, www.artinflanders.be - 124

Figure 11 Matthias de Visch, Marie-Thérèse, 1749-1750, 234 x 149 cm, Inv. Nr. 0000 GRO 0443.I, Hôtel de ville de Bruges, Belgique, Source: Musea Brugge, Lukasweb.be-Art in Flanders, photo: Hugo Maertens, Musea Brugge, www.artinflanders.be - $\mathbf{1 2 5}$

Figure 12 Auteur inconnu, Marie-Thérèse veuve, années 1760, $254 \times 157 \mathrm{~cm}$, autrefois dans le couvent prémontré de Csorna, aujourd'hui au Xántus János Múzeum de Győr, Inv. Nr K.55.185.1, Hongrie, Rómer Flóris Müvészeti és Történeti Múzeum 169

Figure 13 Atelier de Martin van Meytens, Marie-Thérèse, fin des années 1750, $100 \times 70 \mathrm{~cm}$, Inv. Nr. R 455, abbaye de Rein, Autriche - 216 
Figure 14 Atelier de Martin van Meytens, Marie-Thérèse, après 1745, 91 x $71 \mathrm{~cm}$, Inv. Nr. GM 260, salle consacrée à l'empereur de l'abbaye de Klosterneuburg, Stiftsmuseum Klosterneuburg, Autriche - 222

Figure 15 Atelier de Martin van Meytens, François Etienne en habit de cour espagnol, $91 \times 71 \mathrm{~cm}$, Inv. Nr. GM 259, Stiftsmuseum Klosterneuburg, Autriche - 223

Figure 16 Jean-Étienne Liotard, Marie-Thérèse veuve, $74 \times 58 \mathrm{~cm}$, abbaye de Lilienfeld, Autriche, Stiftsarchiv, Stift Lilienfeld. Gemäldesammlung - 225

Figure 17 Atelier de Martin van Meytens, Marie-Thérèse en roi de Hongrie, 150 x 112 $\mathrm{cm}, 1743$, commande des autorités mercantiles de Bolzano, Inv. Nr. 304, Merkantilmuseum Bozen-Museo Mercantile di Bolzano, Italie - 244

Figure 18 Martin van Meytens ou atelier, Marie-Thérèse, début des années 1740, $148 \times 116$ cm, VI 2798a, château de Vizovice, autrefois à Přilepy, en République tchèque, National Heritage Institute, Regional Historic Sites Management in Kroměřiž, State chateau Vizovice - $\mathbf{3 1 0}$

Figure 19 Martin van Meytens ou atelier, Marie-Thérèse, début des années 1740, $119 \times 151 \mathrm{~cm}$, Inv. Nr. A.041, Weinstadtmuseum de Krems, Autriche, museumkrems/Peter Böttcher — 311

Figure 20 Martin van Meytens, Marie-Thérèse, 1744173 x $133 \mathrm{~cm}$, Rollettmuseum de Baden, Inv. Nr. KSP 00235, Rollettmuseum Baden-Städtische Sammlungen Baden, Autriche - $\mathbf{3 1 2}$

Figure 21 Martin van Meytens, portraits de Marie-Thérèse et de François $\left.\right|^{\text {er }}$, vers 1755 , $269 \times 171$ cm, Inv. Nr. G 6987 et 6988 , château de Bruchsal, Spire, Allemagne, staatliche Schlösser und Gärten, Baden-Württemberg, Schloss Bruchsal, photo de l'auteur - 315

Figure 22 Auteur inconnu dans la lignée d'Anton von Maron, Marie-Thérèse, après $1765,84,5$ x 67,5 cm, Inv. Nr. 0 0047, Musée Červený Kameň, Collection of Slovak National Museum-Museum Červený Kameň, Slovaquie — 316

Figure 23 Atelier ou inspiration de Martin van Meytens, Marie-Thérèse, 1742, $103 \times 77,5 \mathrm{~cm}$, collections Esterházy, Inv. Nr. B. 500, Esterházy Privatstiftung, Photo: Manfred Horvath, Schloss Eisenstadt, Autriche - 317

Figure 24 Peintre anonyme, Marie-Thérèse, après 1760 , après $1765,88,5 \times 67,5 \mathrm{~cm}$, Inv. Nr. 0 0128, Musée Červený Kameň, Collection of Slovak national Museum-Museum Červený Kameň, Slovaquie — 318

Figure 25 Martin van Meytens le jeune, Marie-Thérèse, vers 1740-1742, 280 x 184,5 cm, Inv. Nr. NG S 1350, photo de Bojan Salaj, Galerie Nationale de Slovénie, Ljubljana, Slovénie, (c) Narodna Galerija, Ljubljana - 328

Figure 26 Martin van Meytens, Marie-Thérèse, années 1750, 143, 8 x 109, 7 cm, HD 001529 , Musée de la ville de Prague, République tchèque. Collections du Musée. The City of Prague Museum — 343

Figure 27 Atelier de Martin van Meytens, Marie-Thérèse, vers 1760, 154 x $116 \mathrm{~cm}$, Inv. Nr. 1996, Landesmuseum Niederösterreich, @ Landessammlungen NÖ, Photo : Christoph Fuchs, Autriche - 344

Figure 28 Auteur inconnu, Marie-Thérèse, après 1741, probablement dans les années 1750, 150 x 113 cm, Inv. Nr. K. 65. 19. 1, Xántus János Múzeum, Győr, Hongrie, Rómer Flóris Múvészeti és Történeti Múzeum — 347 
Figure 29 Atelier de Martin van Meytens, anonyme, Marie-Thérèse avec la couronne de Bohême, vers 1743, 230 x $151 \mathrm{~cm}$, Galerie nationale de Prague, Inv. Nr. 0 462, République tchèque, Photograph $\odot$ National Gallery Prague $2020-355$

Figure 30 Johann Karl Auerbach, Marie-Thérèse en habit de couronnement de Bohême, 1772, 225 x 150 cm, Inv. Nr. HS 83, Prague Castle Art Collections, République tchèque -368

Figure 31 Cercle d'Auerbach, Marie-Thérèse et Joseph enfant, vers 1745, 231,5 x 150,5 cm, Musée de la ville de Prague, Inv. Nr. H 029 503, Prague, République tchèque. Collections du Musée. The City of Prague Museum - $\mathbf{3 7 0}$

Figure 32 Peter Kobler, double portrait de Marie-Thérèse et de François $\mathrm{I}^{\mathrm{er}}$, 1746, «Stiftsbibliothek St. Florian », 320 x 300 cm, Inv. Nr. 76/12, Sankt Florian, Augustiner Chorherrenstift, Autriche $-\mathbf{3 7 2}$

Figure 33 Martin Johann Schmidt, “Martin Joh. Schmidt fec. 1745”, Marie-Thérèse et l'archiduc Joseph enfant, année 1745, 239 × $157 \mathrm{~cm}$, abbaye de Seitenstetten, Autriche. Le tableau se trouve dans la première pièce de l'abbaye d'été du couvent $-\mathbf{3 9 2}$

Figure 34 Franz Anton Palko, Marie-Thérèse avec ses plus jeunes fils Léopold, Ferdinand et Maximilien, 1766-1768, 445 × $263 \mathrm{~cm}$, autrefois au couvent prémontré de Louka de Znojmo, aujourd'hui au château de Valtice, République tchèque, VA 360, National Heritage Institute Regional Historic sites management in Kroměříž, State château, Valtice - 397

Figure 35 Artiste inconnu, Marie-Thérèse veuve et Joseph II, 50, 6 x $68 \mathrm{~cm}$, château de Kežmarok, Inv. Nr. 10 248, Foto Muzeum v Kežmarku, Slovaquie — 398

Figure 36 Jean-Baptiste ou Jean-Ferdinand Millé, Marie-Thérèse à cheval, 1750 ou années 1750, 315 x 247 cm, Inv. Nr. K 1750/1, Hôtel de Ville de Bruxelles, Musée de la Maison du Roi, Collection des Musées de la Ville de Bruxelles, Belgique - $\mathbf{4 0 2}$

Figure 37 François Eisen, portrait équestre de l'impératrice Marie-Thérèse de Habsbourg, épouse de François Etienne de Lorraine, ancien duc de Lorraine, Huile sur bois, $1757,57,7 \times 43,2 \mathrm{~cm}$, Inv. 72.2.4. ( ) Palais des ducs de Lorraine-Musée Lorrain, Nancy /Photo. G. Mangin, France - 404

Figure 38 Attribué à un peintre autrichien inconnu, Marie-Thérèse en Grand Maître de l'Ordre royal hongrois de Saint-Etienne, seconde moitié du XVIII ${ }^{\mathrm{e}}$ siècle, années 1760, 230 x $151 \mathrm{~cm}$, Inv. Nr. GG_6743, Kunsthistorisches Museum, Gemäldegalerie, KHM-Museumsverband, Vienne, Autriche (C) « KHM-Museumsverband» $\mathbf{4 1 9}$

Figure 39 Anton von Maron, Marie-Thérèse avec la statue de la Paix, 1773, 287 x $125 \mathrm{~cm}$, Inv. Nr. GG_6201, Autriche, Kunsthistorisches Museum, Gemäldegalerie, KHM-Museumsverband, Vienne, Autriche $\odot$ « KHM-Museumsverband » 421

Figure 40 Artiste inconnu, Marie-Thérèse en veuve, $39,5 \times 31 \mathrm{~cm}$, château de Kežmarok, autrefois dans l'hôtel de ville de la cité, Inv. Nr. MK 15 278, Foto Muzeum v Kežmarku, Slovaquie - 423

Figure 41 Peinture non signée, inspiration de Anton von Maron, Marie-Thérèse veuve, années 1770, 117 x 60 cm, Inv. Nr. 0 0301, Musée Červený Kameň, Collection of Slovak National Museum-Museum Červený Kameň, Slovaquie - 424 
Figure 42 Joseph Hickel, Marie-Thérèse veuve, après 1765, 225 x 114 cm, Inv. Nr. 1955/ 15/BI30448, Heeresgeschichtliches Museum, Vienne, Autriche - 425

Figure 43 Attribué au peintre Anton von Maron, mais provient probablement d'un autre peintre, Marie-Thérèse en veuve, vers 1772,136 x 96,5 cm, Inv. Nr. PMPO G-50-s, Musée régional de Ptuj, Pokrajinski muzej Ptuj-Ormož, author: Boris Farič, 2012, Slovénie - 426 